\title{
Laurent Mermet : passeur de frontières négociées pied à pied
}

\author{
Sébastien Treyer*
}

Gestion de l'environnement, Institut du développement durable et des relations internationales, Paris, France

C'est à un partenaire singulier de l'aventure NSS, Laurent Mermet, que nous consacrons cet hommage suite à sa disparition brutale et prématurée l'an passé. Il a été parmi les premiers à s'engager sur la voie alors incertaine que tentait de tracer la revue en offrant une tribune aux chercheurs convaincus que conjuguer sciences et sociétés face aux enjeux environnementaux impliquait le choix de l'interdisciplinarité. Ce que nous rappelle ici son compagnon de route Sébastien Treyer, qui fut aussi son thésard, c'est qu'il fut de tous les combats et de tous les engagements qui ont construit les espaces de réflexion autour des objets et des sciences de l'environnement. Revisiter le parcours de Laurent Mermet, c'est prendre la mesure de la grande diversité d'un champ de recherche et saisir en quoi comprendre pour agir est un appel à l'indiscipline.

La Rédaction

\begin{abstract}
Résumé - Laurent Mermet a construit un parcours intellectuel extrêmement original au sein des communautés de recherche sur l'environnement, autant en France qu'au niveau international, à la croisée entre recherche et action. Centrées sur l'analyse stratégique de la gestion de l'environnement, ses contributions ont animé et alimenté de nombreux collectifs de recherche: sur la concertation et la participation, la négociation, l'évaluation, la prospective, mais aussi au sein de disciplines comme les sciences de gestion, l'économie, l'écologie et la communauté interdisciplinaire que NSS rassemble. Alors qu'il est disparu trop tôt en juin 2019, cet article pointe la diversité et la cohérence de toutes ces contributions, et souligne leur pertinence pour faire face aux crises et transformations en cours dans nos sociétés.
\end{abstract}

Mots-clés : stratégie / évaluation / négociation / prospective / concertation

\begin{abstract}
Laurent Mermet: strategy as a foundational concept to cross and renegotiate boundaries between research communities. Laurent Mermet's research itinerary is both extremely original in the field of environmental studies, and has also provided highly relevant contributions to a diversity of research frontiers, both in France and at the global level. At the boundary between research and action, his work is anchored in the foundational concept of strategy to operate critical analyses on environment management situations, to unveil unsustainability patterns and propose levers for strategic action for change. He has played a key role in various research communities, which this article presents in greater detail: participation, negotiation, evaluation, foresight, as well as in management sciences, economics and ecology. Despite his untimely death in June 2019, his theoretical, conceptual and methodological contributions remain extremely relevant to analyze current crises and transformations in our societies, and to identify pathways for action.
\end{abstract}

Keywords: strategy / participation / negotiation / evaluation / foresight

Parmi les compagnons de route du premier quart de siècle de NSS, Laurent Mermet a construit un itinéraire intellectuel singulier, qui a trouvé une fin brutale avec sa disparition en juin 2019. Lui rendre hommage dans ces pages est à la fois indispensable, vu ses contributions à la

\footnotetext{
*Auteur correspondant : sebastien.treyer@iddri.org
}

revue, mais c'est aussi un moment réflexif important pour repérer les dynamiques collectives en matière de recherches interdisciplinaires sur l'environnement que Laurent Mermet a initiées ou bien auxquelles il a activement contribué, et les pistes qu'elles ouvrent pour appréhender un monde en constante transformation, qu'il nous enjoignait d'analyser avec autant d'enthousiasme 
que de lucidité critique, pour pouvoir toujours mieux y intervenir. Laurent Mermet me manque aujourd'hui beaucoup parce qu'il prenait souvent à contre-pied mes premières hypothèses sur le futur en train de naître sous nos yeux. Il était ainsi un formidable interlocuteur pour mieux comprendre les changements en cours: je peux bien sûr réécouter ses dernières conférences sur sa chaîne YouTube $^{1}$, qui revivifient toutes nos réflexions sur la crise actuelle, dans toutes ses dimensions humaines, sociales, économiques et écologiques, à partir de l'exigence critique qui l'a poussé à délibérément traverser les frontières pour mieux nous questionner. Mais je veux surtout témoigner ici de ses apports fondamentaux à de nombreuses communautés scientifiques. Ayant fait partie de ses premiers thésards à la fin des années 1990, je livre ici ma lecture évidemment personnelle d'un ensemble de collectifs de recherche dont j'ai pu observer combien Laurent Mermet a contribué à les faire progresser, pour en montrer à la fois la diversité, la cohérence, et la pertinence dans le monde d'aujourd'hui.

La traversée de la frontière entre recherche et action est la plus fondatrice de son itinéraire. Toutes les aventures collectives auxquelles Laurent Mermet a participé sont en effet marquées du sceau de la recherche intervention, pratiquée comme une posture doublement exigeante, poussant l'ambition pragmatique dans des retranchements extrêmement féconds autant d'un point de vue scientifique et théorique, que des enseignements pour agir. La distinction entre démarche de recherche et démarche au service de l'action n'est alors pas niée, elle est pratiquée, travaillée, pour générer une dynamique de connaissance et de réinterrogation critique. Le bureau d'études qu'il a fondé en 1988, AScA pour «Application des sciences de l'action», illustre cette ambition par sa mission et par ses réalisations au service des politiques d'environnement ainsi que dans de nombreux programmes de recherche ${ }^{2}$. Il en est ainsi de toutes les autres frontières qu'il a traversées, empiétant pour voir là où ça résiste et pour apprendre autant des divergences que des convergences.

\section{Faire travailler les différences entre situations pour agir}

Le deuxième type de frontières que Laurent Mermet a traversées est tout simplement géographique. Alors que ses recherches sont ancrées dans la compréhension fine

\footnotetext{
1 https://www.youtube.com/channel/UCe7ZcwQSpusCJQ6K 11IEE9g.

${ }^{2}$ Outre les articles auxquels Laurent Mermet a été associé, on trouvera des exemples de l'activité de recherche du bureau d'études AScA dans les pages de NSS: Bouni et al., 2009; Dufour et al., 2017.
}

des spécificités et de l'historicité de l'organisation des systèmes sociaux dans chaque situation et dans chaque territoire particulier, l'aventure intellectuelle qu'il entreprend se joue des frontières. En témoigne son article dans le tout premier numéro de NSS (Mermet, 1993), rendant compte d'un chantier de travail sur les exercices de simulation de politiques au service de la gestion de l'environnement, mené au cours d'un séjour à l'Institut international pour l'analyse des systèmes appliqués (IIASA): dans cet institut, des chercheurs de l'Est et de l'Ouest tirent profit ensemble des dernières avancées de l'analyse systémique, des calculateurs numériques, des recherches en psychologie sociale pour l'appliquer aux grands enjeux systémiques liés aux débats sur les limites de la planète ( $c f$. le rapport du Club de Rome, nommé «rapport Meadows»: [Meadows et al., 1972]) et au développement durable (notamment avec l'ouvrage fondateur de Clark et Munn [1986] souvent éclipsé par le rapport Brundtland [Commission mondiale sur l'environnement et le développement, 1987] - qui pose le premier les principes fondamentaux de la gestion adaptative). C'est dans cet institut que Laurent Mermet participe à la fin des années 1980 à cette effervescence, explore des approches mêlant de manière innovante modélisations intégrées et jeux de rôle, ce dont il rend compte dans cet article de 1993. Il tire des leçons sans concession d'une expérience de recherche participative inspirée des jeux de rôle à destination des états-majors militaires, les wargames, indiquant des lignes-clés de vigilance pour toutes les recherches postérieures mobilisant les jeux de rôle pour la gestion des systèmes socioécologiques, et en particulier l'impossibilité de démêler l'objectif d'apprentissage par les participants et les objectifs de recherche sur les interactions au sein du système socioécologique. Avec cet article, il anticipe également la nécessité de connecter l'espace académique français et francophone de recherche interdisciplinaire sur l'environnement avec les débats les plus avancés en environmental studies.

Entre Est et Ouest, entre espaces francophone et anglophone, Laurent Mermet a aussi été impliqué avec AScA dès les années 1980 dans l'aventure de l'Institut pour une politique européenne de l'environnement, premier think tank au service des politiques européennes d'environnement (aujourd'hui surtout connu sous son acronyme anglais $\operatorname{IEEP}^{3}$ ). Son fondateur Konrad von Moltke avait eu l'intuition que sa pertinence serait d'autant plus grande qu'il saurait se pencher autant sur les politiques publiques et les débats politiques spécialisés des institutions européennes à Bruxelles que sur la spécificité des débats politiques de chacun des Etats membres. C'est exactement dans cette perspective, indispensable pour faire progresser les politiques de

\footnotetext{
3 www.ieep.eu.
} 
durabilité dans l'Union européenne, que travaille l'Iddri (Institut du développement durable et des relations internationales, Sciences Po, Paris) que Laurent Mermet a accompagné comme l'un des membres éminents de son conseil scientifique. L'Iddri est aujourd'hui le membre français d'IEEP, avec plusieurs think tanks européens comme Ecologic ou le Stockholm Environment Institute, avec lequel Laurent Mermet avait déjà collaboré au début des années 1990.

Enfin, en pleine conscience des spécificités historiques des recherches sur l'environnement et le développement dans les pays du Sud, Laurent Mermet a aussi contribué à rapprocher les cadres d'analyse et d'action entre Sud et Nord, tout à fait dans l'esprit qu' inaugurait la conférence de Rio sur l'environnement et le développement en 1992. Accueilli au Cired (Centre international de recherche sur l'environnement et le développement, unité mixte de recherche dirigée à l'époque par JeanCharles Hourcade, et fondée initialement par Ignacy Sachs en lien entre Europe et Brésil, dans l'esprit de l'écodéveloppement), il a lancé, juste après Rio 1992, une série de recherches doctorales qui déploient, dans des contextes de pays en développement, des analyses sur lesquelles nous reviendrons plus loin et sur lesquelles il a lui-même travaillé en France et en Europe. Citons notamment :

- le développement du cadre d'analyse stratégique de la gestion de l'environnement pour l'évaluation des politiques publiques avec les thèses de Maya Leroy (2004 ; gestion des zones humides et du fleuve Sénégal), de Raphaël Billé (2004 ; gestion intégrée des zones côtières en France et à Madagascar) et de Karine Belna (2015 ; mobilisation du fonds partenarial pour le carbone forestier de la Banque mondiale);

- l'analyse des stratégies des acteurs de l'environnement, en particulier des $\mathrm{ONG}$, avec les thèses de Romain Taravella (2008; déforestation en Amazonie brésilienne), Raphaël Billé (2004) et Fanny Guillet (2011 ; protection de la biodiversité des zones humides méditerranéennes en France et au Maroc);

- la prospective avec ma propre thèse (Treyer, 2006; gestion de l'eau en Tunisie et dans l'espace méditerranéen).

Les ouvrages publiés à l'Agence française de développement (AFD), notamment Mermet et Leménager (2015), témoignent de cette volonté de ne pas séparer les enseignements qu'on peut tirer de l'analyse des contextes en développement de ceux qu'on peut tirer de l'analyse des pays développés. C'est bien la proposition au cour de l'Agenda 2030: les 17 Objectifs de développement durable constituent un agenda de transformation profonde de toutes nos sociétés, autant au Sud qu'au Nord, même si la trajectoire de transformation sera inévitablement spécifique pour chacun des pays. Nous devons donc nous employer à identifier des cadres d'analyse et d'action qui permettront de comprendre quels sont les obstacles et les leviers, dans chaque contexte national spécifique, tout en organisant un processus d'apprentissage collectif entre ces différents contextes : c'est dans cette perspective que Laurent Mermet a développé ses analyses, en ne niant pas les différences historiques et géographiques, mais en construisant patiemment des cadres conceptuels permettant de comprendre et d'agir dans les différentes situations. C'est aussi une manière d'exercer un regard critique acéré sur la mondialisation dans ce qu'elle a de plus concret et matériel dans chacun de ces territoires. Nous avons plus que besoin de ce regard critique face à une transformation planétaire qui relie entre eux ces différents contextes, faite de crises comme celle que nous traversons aujourd'hui et de mouvements de fond: une mondialisation en évolution où les rapports géopolitiques et de puissance économique changent vite mais nécessitent d'autant plus une capacité d'analyse critique et stratégique sur ce qui peut constituer la capacité à agir, nœud central du politique.

\section{L'analyse stratégique des situations de gestion, controverse fondamentale avec les autres approches en sciences sociales sur l'environnement}

C'est précisément cette attention à la dimension stratégique de l'agir collectif qui fait toute la saillance du projet intellectuel de Laurent Mermet, en contraste notamment avec ce qu'il a nommé lors d'une de ses dernières conférences en ligne le "refoulé du distributif ». Au centre de son projet intellectuel, l'analyse stratégique des situations de gestion de l'environnement a constitué un travail permanent de différenciation réflexive à la fois pour consolider cette approche ellemême et pousser à la remise en question des principes fondamentaux des autres approches en sciences sociales sur l'environnement, qu'il s'agisse de gestion adaptative des socioécosystèmes, de modélisation d'accompagnement, de gestion patrimoniale, mais aussi des approches sociotechniques, de la théorie de l'acteur réseau ou de la théorie de la justification. Parmi les différentes thèses et articles qui ont présenté, défendu, argumenté les diverses facettes de cette approche, pour qu'elle irrigue l'ensemble des études interdisciplinaires sur l'environnement, c'est l'article dans NSS en 2005 (Mermet et al., 2005) qui constitue le manifeste le plus important pour une mise en débat avec ces différentes communautés de recherche dans l'espace francophone. S'appuyant sur une perspective proche de la sociologie des organisations, 
tout en développant des cadres conceptuels originaux comme l'illustrent les thèses de Maya Leroy (2004), Charlotte Michel (2003) ou Romain Taravella (2008), mettant en usage (et donc aussi en discussion) les travaux de Luc Boltanski et Laurent Thévenot (1991), comme l'illustrent les thèses de Jean Baptiste Narcy (2000) ou de Claire Bouteloup (2015), ces travaux ont alimenté son encadrement de doctorants qui ont essaimé dans différents instituts de recherche et dans des institutions de la politique de l'environnement. Leur point commun est qu'ils mettent l'accent sur la nécessité de réhabiliter une lecture stratégique de situations de gestion, et ce avec plusieurs visées.

Jusqu'alors, au cours des années 1990 qui ont vu naître NSS, de nombreux travaux de recherche avaient cherché à mettre en avant le potentiel de coopération autour de la gestion de l'environnement et des ressources naturelles pour sortir d'une lecture par les structures de domination, explorant ainsi tout le potentiel du concept de «biens communs», notamment sous l'influence des travaux d'Elinor Ostrom. Il s'agissait de ne pas rabattre les enjeux de conflits d'usage autour de ressources rares sur une lecture libérale de tragédie des biens communs et de pouvoir faire exister l'échelle de l'action collective entre celle de l'action publique et l'atomisation des acteurs économiques au sein du marché. Face à cette focalisation sur la coopération, l'analyse stratégique de la gestion environnementale, proposée par Laurent Mermet, vise à remettre au cœur de la réflexion la compétition au moins autant que la coopération, les antagonismes au moins autant que les synergies. Pour le dire simplement, beaucoup de travaux ont présenté l'action collective pour préserver l'environnement avant tout comme un problème de coordination ou de manque d'information ou de connaissances des positions et perceptions des autres acteurs : aujourd'hui encore, c'est cette dimension de coordination qui est la plus présente dans les discours politiques, voire académiques, sur la mise en œuvre des objectifs de développement durable, et dans le concept de gouvernance lui-même. Les obstacles à la transition écologique mettent pourtant aujourd'hui très clairement en évidence qu'on ne trouvera pas le chemin sans penser ensemble les dimensions de coordination et de compétition ou de conflit. Laurent Mermet a en particulier insisté sur le rôle central des acteurs minoritaires de changement, et sur la nécessité de les équiper d'outils d'analyse stratégique pour que le changement puisse avoir lieu plutôt qu'il ne soit noyé dans les résistances, les verrouillages ou des formes d'intégration des enjeux environnementaux trop partielles pour conduire à une véritable transformation. Le champ de la sociologie de l'innovation a offert avec le concept de régime sociotechnique un vocabulaire permettant de penser ensemble les obstacles au changement, tant dans le champ des résistances politiques que des verrouillages sociotechniques (Geels et Schot, 2007). L'approche stratégique proposée par Laurent Mermet n'est ainsi plus la seule à tenter explicitement de trouver des moyens d'agir face au caractère inséparable de ces deux dimensions de l'action collective. Elle reste cependant essentielle comme repère d'analyse critique ou comme antidote pour éviter de s'aveugler sur une des deux faces, pourtant toujours présentes, de toute situation de gestion: en particulier, elle nous apprend encore aujourd'hui à éviter de refouler le dissensus et le conflit pour pouvoir s'en saisir comme une dimension à analyser et avec laquelle jouer pour agir.

Deuxièmement, il s'agit aussi de puiser dans tout un champ de littérature peu connecté aux approches interdisciplinaires en matière d'environnement: celui de la recherche en stratégie, depuis les grands auteurs classiques comme Thucydide ou Sun Tzeu, et qui, en sciences de gestion, se centre surtout sur les organisations formelles que constituent les entreprises; mais aussi celui de la rhétorique, concrétisation majeure de la stratégie dans l'espace discursif lié aux processus des politiques publiques.

Troisièmement, avec l'analyse stratégique de la gestion de l'environnement, Laurent Mermet revendique la possibilité et la nécessité d'une recherche ancrée dans une perspective normative explicite, celle de la quête de l'efficacité en matière environnementale. Cette caractéristique de ses travaux l'a souvent positionné au cœur de controverses, en ce qu'il interrogeait les paradoxes fondamentaux de postures de recherche qui, à l'inverse, optent pour une axiomatique neutre ou visent des résultats purement procéduraux. Cette posture épistémologique a des convergences fortes avec les travaux de Michel Callon ou Bruno Latour (Callon et Latour, 1991), qui ont montré combien toute activité scientifique procède d'une forme de normativité, souvent implicite, parfois plus explicite. Pour Laurent Mermet, il est indispensable que des chercheurs tiennent de manière tout à fait transparente et revendiquée une posture d'évaluation de l'efficacité de l'action à partir d'un point de vue normatif particulier, pour expliciter les enjeux des situations de gestion vues depuis la perspective des acteurs en charge de la protection de l'environnement: une telle posture est aussi un formidable levier critique pour analyser nos sociétés et leurs transformations. C'est dans cet esprit qu'il a développé le concept de recherche environnementale sur la société, essentiellement sous forme d'une série de vidéos, accessibles en ligne sur sa chaîne YouTube ${ }^{4}$. Il explicite ce projet avant tout comme une contribution critique indispensable dans un moment où la non-durabilité des évolutions tant économiques que sociales ou environnementales se fait particulièrement

\footnotetext{
${ }^{4}$ https://www.youtube.com/channel/UCe7ZcwQSpusCJQ6K 11IEE9g.
} 
sentir. Dans cette série de conférences, il met aussi l'accent sur la perspective d'une philosophie politique ancrée dans le pluralisme des préoccupations (matters of concern, dirait Bruno Latour; voir Latour, 2004) qui doivent être portées de manière stratégique autant par des acteurs que par des chercheurs pour pouvoir s'approcher d'une formation de l'intérêt général. Refusant l'injonction que le chercheur doive lui-même faire l'intégration de ces différentes préoccupations dans ses travaux, jugeant impossible une recherche en surplomb de la société, cette posture crée un dissensus fertile avec d'autres écoles de pensée. En particulier, malgré les convergences indiquées plus haut avec la sociologie des sciences, il a aussi particulièrement souligné sa différence de perspective avec Bruno Latour, tant sur la question de « comprendre » que celle d' «agir », entre un acteur qui lit le réseau d'actants et d'acteurs en s'y immergeant, acceptant les biais d'une telle situation, et le chercheur qui cherche à s'en extraire pour observer ce réseau de l'extérieur, peinant en revanche symétriquement à identifier des leviers politiques susceptibles de faire changer le cours des tendances à la dégradation de l'environnement. Laurent Mermet a également poursuivi ces débats sur sa chaîne YouTube, s'inspirant d'une actualité particulièrement vivante en 2018 avec la démission de Nicolas Hulot du ministère de la Transition écologique et solidaire ou le mouvement des gilets jaunes, faisant vivre sa posture d'universitaire engagé dans la controverse politique là où elle lui semblait la plus vive, notamment pour entrer en discussion critique autant sur les contradictions implicites de la mobilisation pour l'action chez les collapsologues que sur l'impossibilité d'arbitrages politiques ambitieux à l'échelle nationale, à la faveur du débat ouvert par la démission de Nicolas Hulot.

Laurent Mermet a ainsi engagé une discussion permanente avec les communautés de recherche que NSS rassemble, animé par une volonté de différencier et de contraster son approche pour ouvrir un débat sans fard, et notamment dans plusieurs numéros de NSS, comme en témoignent les références mobilisées dans cet article. Les recherches critiques sur les organisations et la stratégie constituent une communauté spécifique au sein des sciences de gestion, et son approche de la critique environnementale de nos sociétés a trouvé des relais grâce aux travaux de Maya Leroy, au sein de Montpellier Recherche en Management (MRM) et dans des réseaux internationaux de recherche en sciences de gestion qui lui sont liés, ce qui constitue un des développements les plus notables de ce champ de recherche.

Dans le domaine de l'action publique, la traduction concrète de cette aventure scientifique a été permise par le recrutement de Laurent Mermet comme professeur à l'École nationale du génie rural, des eaux et des forêts (aujourd'hui composante d'AgroParisTech, au sein de
l'Université Paris-Saclay), école d'application formant les hauts fonctionnaires du ministère français de l'Agriculture. Il y a contribué non seulement aux enseignements mais aussi à une véritable révolution au sein de ce corps d'ingénieurs de l'État, apportant une réflexion critique ou, selon ses propres termes, une forme de subversion d'une «administration agricole » des idées et de l'action publique. Celle-ci est façonnée par l'expérience de la cogestion de la politique agricole et marquée par ses difficultés à réformer cette politique en France et en Europe. Laurent Mermet a introduit dans les formations des ingénieurs des secteurs de l'agriculture, de l'équipement et de l'environnement une pensée de la dialectique entre une préoccupation environnementale, qui devrait se concevoir davantage comme un secteur d'action publique et pas uniquement comme étant en charge d'une mission intégratrice entre secteurs, et des organisations sectorielles de l'action publique particulièrement solides, résilientes, voire résistantes au changement ou faisant l'objet de verrouillages sociotechniques. L'influence de Laurent Mermet à cet égard est difficile à mesurer, mais la présence de ses anciens élèves dans diverses administrations et agences est tout à fait notable, et les formations qu'il a contribué à créer à AgroParisTech (mastère spécialisé «Politiques publiques et stratégies pour l'environnement ») ou auxquelles il a participé (mastère spécialisé «Forêt, nature et société - Management international », domaine d'approfondissement du cursus d'ingénieur d'AgroParisTech en gestion environnementale des écosystèmes et forêts tropicales) constituent un capital important qu'il convient de soutenir et de développer.

\section{Concertation, négociation, évaluation, prospective: quatre champs de recherche où la figure de Laurent Mermet est indispensable au collectif}

À partir de cet ancrage dans l'analyse stratégique de la gestion de l'environnement, où il s'est posé dans une logique de différenciation réflexive, Laurent Mermet a aussi activement participé à plusieurs autres communautés de recherche, où ses apports ont permis et permettent encore des avancées du front des questions et des analyses critiques.

Première d'entre elles, la recherche sur la participation et la concertation. Laurent Mermet a été président du conseil scientifique du programme «Concertation, décision, environnement $» d u$ ministère en charge de l'environnement (service de la recherche et de la prospective), qui a rythmé par ses appels à propositions de recherche et ses colloques la communauté scientifique française sur ces questions de 1998 à 2013. Avec Denis Salles, aujourd'hui directeur de recherche à INRAE, il a 
dirigé en 2015 l'ouvrage collectif qui tire le bilan de toute cette aventure (Mermet et Salles, 2015). Ce programme a notamment accompagné à la fois l'essor et la multiplication des dispositifs participatifs au service de l'action environnementale et du développement durable, mais aussi le regain de postures critiques sur ces démarches, et l'irruption de formes d'agir collectif et de mobilisations sociales non nécessairement organisées en mécanismes d'action publique. Dans cette même perspective, il a également été membre du comité d'observance (compliance committee) de la convention d'Aarhus sur l'accès à l'information, la participation du public et l'accès à la justice en matière d'environnement, dans le cadre de la Commission économique pour l'Europe des Nations unies. Aujourd'hui, alors que les contestations sociales et les mobilisations politiques secouent nos démocraties aux prises avec les crises sociales et écologiques, des expériences d'échelle inédite de participation explorent les modalités permettant de complémenter la démocratie représentative, comme la convention citoyenne pour le climat. Les concepts de Laurent Mermet sont essentiels pour en faire l'analyse critique et leur donner du sens.

Autre communauté très proche, celle des recherches sur la négociation. C'est tout naturellement que Laurent Mermet a joué un rôle-clé dans cette communauté, à la croisée des sciences de gestion, de la sociologie, de l'économie et des sciences politiques: le recadrage principal, qu'il a proposé pour analyser les processus de concertation et de participation, est de les considérer comme des moments spécifiques au sein d'un processus plus large et plus long de négociation entre acteurs. La négociation est d'ailleurs un concept-clé dans son projet intellectuel, puisqu'elle propose un cadre conceptuel qui vise à analyser les diverses formes de coexistence de la dimension coopérative et de la dimension distributive en matière d'action collective. La revue Négociations (2019), à laquelle il a participé activement, lui a d'ailleurs rendu un très bel hommage, mentionnant son engagement au sein du groupe international PIN (Processes of international negotiation) et du Groupe français de la négociation. On pourrait également classer dans cette proximité entre concertation, négociation et son intérêt initial pour les jeux de rôle, ses réflexions sur le jeu, inspiré par exemple par Homo ludens de Huizinga (1951), ce qui l'a conduit à animer un colloque de Cerisy sur le thème du jeu avec Nathalie Zaccaï-Reyners en $2013^{5}$. Une démarche tout à fait illustrative de sa pensée qui procède par un retour aux fondamentaux conceptuels

\footnotetext{
${ }^{5}$ Le programme du colloque est disponible à cette adresse : http://www.ccic-cerisy.asso.fr/jeu13.html. Un ouvrage en est issu (Mermet et Zaccaï-Reyners, 2015).
}

d'une notion comme le jeu, pour revenir irriguer les recherches et l'action elle-même.

Troisième communauté de recherche, celle sur l'évaluation des politiques publiques, comme une forme d'expertise particulière. Souvent difficile à classer en France, elle se situe à l'interface entre consultance et recherche, et elle est pourtant essentielle dans les recherches sur l'environnement. Les travaux de Laurent Mermet sur la politique de protection des zones humides en France ont été cruciaux pour fonder son analyse sur ces sujets. Il en a rendu compte en 1997 dans les pages de NSS (Mermet et Barnaud, 1997). La thèse de Maya Leroy (2004) sur la gestion du fleuve Sénégal et celle de Raphaël Billé (2004) sur la gestion intégrée des zones côtières constituent également des avancées majeures dans ce champ. Elles rejoignent notamment la lecture de l'évaluation des politiques publiques comme intervention stratégique, que l'on retrouve comme une des perspectives sur l'évaluation présentées dans l'ouvrage coordonné par Pieter Leroy et Ann Crabbé (2008). Alors que les travaux sur la mise en œuvre du développement durable aboutissent trop souvent au constat du manque de cohérence des politiques publiques, qu'il conviendrait de mieux intégrer ( « sortir des silos »), Laurent Mermet et ses collègues proposent de ne pas nier l'inévitable constitution des organisations humaines sous forme de secteurs indépendants, et au contraire de s'appuyer sur une lecture critique et stratégique des processus d'élaboration et de mise en œuvre des politiques publiques comme des négociations entre secteurs. L'évaluation de l'incohérence des politiques publiques devient alors un levier stratégique au service du changement. Comme pour les autres communautés de recherche mentionnées ci-dessus, Laurent Mermet est intervenu autant auprès des chercheurs francophones que dans les communautés académiques d'échelle internationale, très anglophones en matière d'évaluation des politiques publiques, notamment avec un article manifeste (Mermet et al., 2010).

Quatrième communauté de recherche que Laurent Mermet a fortement contribué à renouveler, celle sur la prospective et, notamment, dans un champ des recherches sur l'environnement où les travaux prospectifs se sont multipliés depuis les années 1990, parfois sans en porter le nom (scénarios à 2050 ou 2100 des impacts du changement climatique, par exemple). Dans le champ de la prospective en général, dont la dimension narrative, créative et ludique, a probablement été une des sources de sa curiosité à son égard, il a notamment introduit une perspective stratégique, où chaque démarche prospective, au lieu d'être vue seulement comme une combinaison d'outils à mettre en œuvre, devait avant tout être considérée comme une intervention stratégique au service d'un projet de changement, comme l'ont souligné les thèses qu'il a fait soutenir (Van der Helm, 
2005 ; Treyer, 2006; Labbouz, 2014). Cette perspective stratégique rejoint aujourd'hui d'autres champs de recherche qui présentent la manufacture des futurs comme un champ de bataille stratégique, ou pour le moins un champ de forces, comme dans l'exemple du concept de promesses technoscientifiques (du champ des science and technology studies) qui souligne la capacité de certaines promesses à s'imposer comme dominantes en réussissant à exclure d'autres options et scénarios possibles. Un numéro spécial de la revue Futures, principale revue internationale en matière de prospective, rend compte de cette proposition de renouvellement conceptuel du champ (Mermet et al., 2009; Mermet, 2009). Plus spécifiquement, au sein des recherches sur l'environnement, Laurent Mermet a participé à l'effort de révélation de la dimension proprement prospective de nombreux travaux de recherche sur les scénarios futurs de dégradation de l'environnement ou de transition, qu'il s'agisse de modélisations numériques ou d'exercices participatifs prospectifs: il a notamment appelé la communauté des recherches sur l'environnement à se constituer comme un forum prospectif de mise en discussion de ces scénarios, objets toujours hybrides entre description d'un monde possible et incitation à agir. Dans le cadre du programme «Environnement, vie et sociétés » du CNRS, une école chercheurs organisée en octobre 2001 a été fondatrice de cette réflexivité au sein de la communauté française de recherche, ce dont témoigne un article paru dans NSS (Mermet et Poux, 2002). Les bases extensives de cette réflexion sur les recherches prospectives pour l'environnement ont ensuite été posées dans l'ouvrage collectif Étudier des écologies futures. Un chantier ouvert pour les recherches prospectives environnementales (Mermet, 2005). On pourrait aussi ajouter la diversité des fils que cette perspective de recherche lui permettait de tirer du côté de la rhétorique, de la construction narrative, de l'argumentation, car ce sont là d'autres voies extrêmement utiles qu'il nous reste encore à explorer.

Enfin, il faut aussi souligner l'inscription de Laurent Mermet au sein des sciences de gestion, en tant que discipline pleinement alignée sur la posture de rechercheintervention qui a caractérisé la plupart de ses travaux. Leur portée fondamentalement critique sur les transformations en cours dans nos sociétés entrait en résonance très forte avec les critical management studies, qui se situent au cœur du système de la pensée managériale, à la fois pour saisir toute la force de cette réflexion sur l'action collective, et aussi pour pouvoir la critiquer de l'intérieur. Ce parcours de Laurent Mermet est poursuivi aujourd'hui par l'équipe Montpellier Recherche en Management, sous la houlette de Maya Leroy, ainsi qu'à travers les travaux de thèse conduits par Clément Feger dans le champ des «critical accounting studies» (Feger, 2016). Ces recherches ont conduit à un dialogue approfondi avec la communauté internationale de recherche sur le capital naturel (Feger et al., 2018) et à la proposition de nouvelles formes de comptabilités pour la gestion des écosystèmes (Feger et Mermet, 2017).

Les sciences de gestion sont aussi une discipline carrefour, permettant des liens avec de nombreuses autres disciplines : la sociologie des organisations, l'économie (d'où le rattachement de Laurent Mermet au Cired [Centre international de recherche sur l'environnement et le développement]), la science politique (d'où son rattachement au Cerses [Centre de recherche sens, éthique et société]), mais aussi et surtout l'écologie, qui lui tenait particulièrement à cœur et qui était sa formation initiale (d'où son rattachement au Cesco [Centre d'écologie et des sciences de la conservation]). Ce parcours au sein de collectifs disciplinaires divers, qui a contribué à faire de lui un inclassable, l'a mené à participer activement à des dynamiques collectives au sein de chacune de ces disciplines. On pourrait mentionner - de manière trop rapide - les ateliers de recherche sur la prospective en écologie, menés autour de la thèse d'Audrey Coreau (2009), et qui sont un des exemples les plus saillants d'une conversation soutenue avec l'écologie de la conservation (Mermet, 2018); ou encore - et c'est un hommage qu'il faut rendre ici à Claude Henry, son directeur de thèse (Mermet, 1989) - des avancées fondamentales pour l'économie de l'environnement: dans la droite ligne des travaux de Claude Henry sur l'économie comme langage et enjeu de négociations, Laurent Mermet a participé à changer la perspective sur les évaluations économiques des services écosystémiques en questionnant leur utilisation réelle dans les processus de décision, avec la publication d'articles qui ont fait franchir une étape décisive à cette communauté de recherche (Laurans et al., 2013; Laurans et Mermet, 2014).

Viscéralement attaché au pluralisme, Laurent Mermet a donc fortement contribué à pluraliser les recherches elles-mêmes et les postures de recherche au sein d'une grande diversité de communautés scientifiques, en particulier celles dont nous avons besoin aujourd'hui pour donner du sens aux transformations que nous subissons ou que nous cherchons à faire advenir. Son regard et ses concepts fondamentalement critiques nous accompagnent et sont d'une utilité essentielle pour aborder le monde en train de bouger.

\section{Références}

Belna K., 2015. La protection de la biodiversité, "cobénéfice" évident ou point de tension central des programmes REDD+ élaborés en vue d'atténuer le changement climatique lié à la déforestation des forêts tropicales?. Thèse de doctorat en sciences de l'environnement, Paris, AgroParisTech. 
Billé R., 2004. La gestion intégrée du littoral se décrète-telle? Une analyse stratégique de la mise en ouvre, entre approche programme et cadre normatif. Thèse de doctorat en sciences de l'environnement, Paris, ENGREF.

Boltanski L., Thévenot L., 1991. De la justification. Les économies de la grandeur, Paris, Gallimard.

Bouni C., Chevillotte G., Dufour A., 2009. Indicateurs du développement durable des transports et concertation avec le public: postures stratégiques et argumentations, Natures Sciences Sociétés, 17, 4, 339-348, https://doi.org/10.1051/ nss/2009058.

Bouteloup C., 2015. Agir pour la reconnaissance du dommage écologique des marées noires: attachements, stratégies et justification. Cas de l'Amoco Cadiz et de l'Erika. Thèse de doctorat en sciences de l'environnement, Paris, AgroParisTech.

Callon M., Latour B. (Eds), 1991. La science telle qu'elle se fait. Anthologie de la sociologie des sciences de langue anglaise, Paris, La Découverte.

Clark W.C., Munn R.E. (Eds), 1986. Sustainable development of the biosphere, Cambridge, Cambridge University Press.

Commission mondiale sur l'environnement et le développement, 1987. Notre avenir à tous, Organisation des Nations unies. Traduit de: Our common future, United Nations.

Coreau A., 2009. Dialogue entre des chiffres et des lettres: imaginer et construire des futurs possibles en écologie. Thèse de doctorat en écologie, Montpellier, Université Montpellier 2.

Dufour A., Narcy J.-B., Raoul Duval J., 2017. La recherche finalisée, une approche ouverte sur la singularité des terrains, Natures Sciences Sociétés, 25, S29-S35, https:// doi.org/10.1051/nss/2017044.

Feger C., 2016. Nouvelles comptabilités au service des écosystèmes. Une recherche engagée auprès d'une entreprise du secteur de l'environnement. Thèse de doctorat en sciences de gestion, Paris, AgroParisTech.

Feger C., Mermet L., 2017. A blueprint towards accounting for the management of ecosystems, Accounting, Auditing and Accountability Journal, 30, 7, 1511-1536, https://doi.org/ 10.1108/AAAJ-12-2015-2360.

Feger C., Mermet L., Vira B., Addison P.F.E., Barker R., Birkin F., Burns J., Cooper S., Couvet D., Cuckston T., Daily G.C., Dey C., Gallagher L., Hails R., Jollands S., Mace G., Mckenzie E., Milne M., Quattrone P., Rambaud A., Russell S., Santamaria M., Sutherland W.J., 2018. Four priorities for new links between conservation science and accounting research, Conservation Biology, 33, 4, 972-975, https://doi. org/10.1111/cobi.13254.

Geels F.W., Schot J., 2007. Typology of sociotechnical transition pathways, Research Policy, 36, 3, 399-417, https://doi.org/10.1016/j.respol.2007.01.003.

Guillet F., 2011. Une analyse stratégique pour l'organisation à finalité environnementale: le cas d'une ONGE, la Tour du Valat, Centre de recherche pour la conservation des zones humides méditerranéennes. Thèse de doctorat en sciences de gestion, Paris, AgroParisTech.
Huizinga J., 1951. Homo ludens. Essai sur la fonction sociale du jeu, Paris, Gallimard.

Labbouz B., 2014. Sécurité alimentaire et futurs de l'agriculture mondiale. Comprendre un forum prospectif international en émergence et réfléchir aux façons d'y intervenir. Thèse de doctorat en sciences de l'environnement, Paris, AgroParisTech.

Latour B., 2004. Why has critique run out of steam? From matters of fact to matters of concern, Critical Inquiry, 30, 2, 225-248, https://doi.org/10.1086/421123.

Laurans Y., Mermet L., 2014. Ecosystem services economic valuation, decision-support system or advocacy?, Ecosystem Services, 7, 98-105, https://doi.org/10.1016/j.eco ser.2013.10.002.

Laurans Y., Rankovic A., Billé R., Pirard R., Mermet L., 2013. Use of ecosystem services valuation for decision making: questioning a literature blindspot, Journal of Environmental Management, 119, 208-219, https://doi.org/10.1016/j.jenv man.2013.01.008.

Leroy M., 2004. Gestion stratégique des écosystèmes dans un contexte d'aide internationale: engagements environnementaux et dispositifs de gestion dans la vallée du fleuve Sénégal. Thèse de doctorat en sciences de l'environnement, Paris, ENGREF.

Leroy P., Crabbé A., 2008. Handbook of environmental policy evaluation, London, Earthscan.

Meadows M., Meadows D., Randers J., Behrens W.W. III., 1972. The limits to growth. A report for the Club of Rome's Project on the predicament of mankind, New York, Universe Books. Trad. fr. : Halte à la croissance?, Paris, Fayard.

Mermet L., 1989. La nature, jeu de société: une analyse stratégique pour la gestion de l'environnement. Thèse de doctorat en sciences de gestion, Paris, Université Paris 9.

Mermet L., 1993. Une méthode de prospective : les exercices de simulation de politiques, Natures Sciences Sociétés, 1, 1, 34-46, https://doi.org/10.1051/nss/19930101034.

Mermet L., 2005. Étudier des écologies futures. Un chantier ouvert pour les recherches prospectives environnementales, Peter Lang.

Mermet L., 2009. Extending the perimeter of reflexive debate on futures research: an open framework, Futures, 41, 2, 105-115, https://doi.org/10.1016/j.futures.2008.07.044.

Mermet L., 2018. Knowledge that is actionable by whom? Underlying models of organized action for conservation, Environmental Science \& Policy, 113, 39-46, https://doi. org/10.1016/j.envsci.2018.04.004.

Mermet L., Barnaud G., 1997. Les systèmes de caractérisation des zones humides: construire l'expertise sous pression politique, Natures Sciences Sociétés, 5, 2, 31-40, https://doi. org/10.1016/S1240-1307(97)86197-3.

Mermet L., Billé R., Leroy M., 2010. Concern-focused evaluation for ambiguous and conflicting policies: an approach from the environmental field, American Journal of Evaluation, 31, 2, 180-198, https://doi.org/10.1177/1098214010366047.

Mermet L., Billé R., Leroy M., Narcy J.-B., Poux X., 2005. L'analyse stratégique de la gestion environnementale: un 
cadre théorique pour penser l'efficacité en matière d'environnement?, Natures Sciences Sociétés, 13, 2, 127-137, https://doi.org/10.1051/nss:2005018.

Mermet L., Fuller T., Van der Helm R., 2009. Re-examining and renewing theoretical underpinnings of the futures field: a pressing and long-term challenge, Futures, 41, 2, 67-70, https://doi.org/10.1016/j.futures.2008.07.040.

Mermet L., Leménager T. (Eds), 2015. Développement et biodiversité: comment négocier le tournant environnemental?, Paris, Agence française de développement.

Mermet L., Poux X., 2002. Pour une recherche prospective en environnement: repères théoriques et méthodologiques, Natures Sciences Sociétés, 10, 3, 7-15, https://doi.org/ 10.1016/S1240-1307(02)80164-9.

Mermet L., Salles D. (Eds), 2015. Environnement: la concertation apprivoisée, contestée, dépassée?, Louvainla-Neuve, De Boeck.

Mermet L., Zaccaï-Reyners N. (Eds), 2015. Au prisme du jeu. Concepts, pratiques, perspectives, Paris, Hermann.

Michel C., 2003. L'accès du public aux espaces naturels, agricoles et forestiers et l'exercice du droit de propriété: des équilibres à gérer. Thèse de doctorat en sciences de l'environnement, Paris, ENGREF.
Narcy J.-B., 2000. Les conditions d'une gestion spatiale de l'eau. Le monde de l'eau face aux filières de gestion des espaces. Thèse de doctorat en sciences et techniques, Paris, ENGREF.

Négociations, 2019. In memoriam. Laurent Mermet (1955-2019), Négociations, 32, 2, 5-10, https://doi.org/10.3917/ neg.032.0005.

Taravella R., 2008. La frontière amazonienne aujourd'hui : projet socio-environnemental de conservation forestière contre dynamique pastorale de déforestation. Une analyse stratégique 2000-2006 de l'action collective en "Terra do Meio» (Para, Brésil). Thèse de doctorat en sciences de l'environnement, Paris, AgroParisTech.

Treyer S., 2006. À quelle raréfaction de l'eau faut-il se préparer? Construire une intervention prospective au service de la planification pour les ressources en eau en Tunisie. Thèse de doctorat en sciences de l'eau, Paris, ENGREF.

Van der Helm R., 2005. Le phénomène vision. Une enquête sur la nature et sur le fonctionnement des "visions du futur" et sur leurs usages pour la gestion de l'environnement. Thèse de doctorat en sciences de l'environnement, Paris, ENGREF.

Citation de l'article : Treyer S. Laurent Mermet: passeur de frontières négociées pied à pied. Nat. Sci. Soc. 28, 2, 169-177. 